\title{
DRAFT
}

\section{Distribution System of the Future}

John D. Kueck

Brendan J. Kirby

Oak Ridge National Laboratory

\section{Introduction}

The distribution system of the future is going to be as much of a revolution to the electric energy industry as the wireless telephone has been to consumer communications. An electricity market transformation must occur before the changes can take place, but this evolution is already starting to occur in many parts of the country. In this paper, we discuss a vision for a future distribution system, areas that will be key for technology development, and the advantages of the new electricity market.

Present day distribution systems are in a sense, unintelligent. Distribution systems respond to faults, or short circuits, by sensing the very high fault current and then opening circuit breakers to isolate the fault. Some newer automated systems determine fault location and then close other circuit breakers to provide an alternate path for power after the fault so that the number of customers left without power is minimized, but the extent of the reconfiguration is limited. Distribution systems also have some methods to regulate voltage, but there is little real time local response to contingencies such as loss of a transmission line or a generator.

In present day distribution systems, there is very little control of load, or demand response, and Distributed Energy Resources (DER, distributed generation, storage, and responsive load) located in the distribution system are prohibited from even regulating voltage. In fact, industry standards and utility interconnection agreements typically require that when a contingency occurs on a distribution or transmission system that results in a voltage or frequency excursion, the DER is to disconnect rather than help.

There is a pressing need to evolve the distribution system model to one that can respond to contingencies sensed locally, and has the local intelligence and autonomy to deal with contingencies such as unusual loading, transmission congestion, and line outages.

Markets must be simple for customers to participate in the energy and reliability services transactions.

In the future, distribution systems will have local monitors for current, voltage, and temperature at many locations such as substations, underground cable and transformer vaults, feeders and laterals. These monitors will input data to local agents. The local agents will be computers scattered throughout the distribution system constantly assessing system condition. The local agents will communicate quickly with their local 


\section{DRAFT}

sensors and with each other, and more slowly with status reports they send to the central control authority. The local agents will review temperature, current and voltage data and diagnose problems such as overloads, over/under voltages, and voltage presence on circuits that are supposed to be deenergized. They will also sense contingencies such as equipment outage due to faults.

The local agents will be partially independent from central control and authorized to take corrective action when they diagnose problems. Some of the actions that will be taken will be local dispatch of DER, local capacitor connection, voltage regulator control, local circuit switching, and provision of local reliability services, such as load response. The local agents will report up to a central agent on a periodic basis. There will be several different types of local agents that will function together.

One of the most important capabilities will be for the customers to participate in the energy and reliability services markets. With only a few exceptions, these markets are now only open to large generators and a few large loads. Reliability services, such as voltage regulation or reserve power, are much more effective when supplied locally at the load. In addition, local participation in these markets will provide demand elasticity in the load - something that is lacking now. At present, when power is scarce due to a contingency or severe weather, there is no customer response; customers continue to use the same amount because they do not see the market price. An elastic demand means that some customers would cut back during periods of shortage and high price. Elasticity in demand is essential for a healthy market system.

\section{Vision for the Future}

\section{Increased level of safety for linesmen.}

Presently, linesmen rely on the central control operator to remotely open circuit breakers and clear circuits. In some instances, the linesmen will open disconnects and pull fuses themselves to isolate equipment. These precautions will also be used in the future, but there will also be a backup level of monitoring and protection provided by the local agents. The local agents will be notified of utility personnel working on a circuit, they will be involved in the de-energization of the circuit, and they will ensure that the circuit is kept de-energized to provide a secondary level of protection. This secondary level will ensure that DER is not connected, or other switching is not performed which could energize the equipment. The local agents will also monitor the equipment to be sure that it has no voltage.

\section{Reduced electrical losses and minimized damage due to faults.}

Presently, faults are detected by protective relaying and cleared by operation of circuit breakers or fuses. Protective relaying and fuses both operate by detecting abnormal levels of current flow. In the future, abnormal current flows will also be detected, but in addition to conventional methods, techniques will be used that evaluate the signature of 


\section{DRAFT}

the current to determine the presence of an arcing fault, high resistance ground fault, faults in the presence of DER, and other fault types which are difficult to detect today. In addition, fault protection will act much faster, static switches will operate much more quickly than circuit breakers, and fault currents will be cleared much more quickly. This will result in greatly reduced equipment damage due to faults and shortened voltage sag events during fault clearing.

Automatic fault detection and location methods can quickly isolate the faulted equipment and restore power to unfaulted sections in fractions of a second. Outage times and areas can be greatly reduced. In addition, fault detection and isolation on equipment fed from power electronic devices can limit fault current in fractions of a cycle. Such short durations of fault current mean that damage from the fault current will be minimal. At the present time, motor damage from faults in motors fed from adjustable speed drives has been essentially eliminated. The drive detects the fault and interrupts so quickly that only the fault point is damaged. There is no secondary winding or rotor damage from arcing or sustained over-current or high levels of fault current. This same advantage can be present in the distribution system.

\section{Advantages in Predictive Maintenance}

Local agents will analyze currents for symptoms of transformer, circuit breaker, cable, and other equipment degradation, sending signals up to the central control when degradation is detected. This assessment will be made automatically by analysis of current, voltage, vibration, temperature and other signals. The agents will be equipped with analysis software to assess the condition of the equipment in their local area. Analysis techniques are being developed now for specific equipment such as cable, transformers and circuit breakers. These techniques will be much more powerful when used to assess equipment condition in a systematic, automated, mechanism. Maintenance can then be planned in advance of equipment failure so that equipment such as transformers or circuit breakers may be kept in service until an appropriate time is available.

\section{System Automation and Autonomy}

Central control will only provide overview instructions to the local agents. Under normal conditions, these instructions will be voltage schedules and default system configurations. The local agents will be responsible for regulating voltage within the schedule using local sources of reactive power, generation, load tap changers and other methods. The local agents will also be responsible for dealing with routine contingencies such as line faults and transformer failures. Local agents will also have responsibility for reconfiguring feeders to maintain service after faults. This is done now to a limited extent, in the future it will include reconfigurable islands. The local agents will periodically update the central controller on the status of local system operation. High speed data transmission will be available both up to the central operator and down to the local agent for reports or commands dealing with emergency issues such as system failure and islanding 


\section{DRAFT}

\section{Local Provision of Reliability Services}

One of the most exciting prospects of the distribution system of the future will be its ability to provide ancillary (reliability) services. These services will be supplied in response to market signals. The services may be contracted over the internet. Both loads and DER will supply these services. Supplying the services locally is many times more efficient than supplying them from distant generating stations. In addition to an intelligent distribution system, an automated market system will also be necessary to make this happen.

\section{Reduced cost, both capital and operations and maintenance.}

Feeder configuration will be monitored in real time to ensure that circuits are configured to minimize losses, and local reactive power is supplied to optimize voltage and minimize reactive power flow. Real time monitoring and control of transformer tap position, generator voltages and phase angles and VAR sources have yielded loss reductions of nearly $40 \%$ in transmission systems while other operational constraints are still satisfied. [1] It is not unreasonable to expect that similar improvements could be realized in distribution systems. Fraud will be addressed as well. Power flow will be monitored through the substation, distribution feeders, laterals, distribution transformers and secondaries. Any anomalies such as points with high losses will be detected. It will also be possible to compare revenue meters with measured power delivered to secondaries.

\section{Information Technology}

As wires companies move further into restructuring, they will be operating at higher and higher levels of business and operational efficiency. Their information technology needs will grow as the need to compete grows. Information will be used for customer service and for providing such features as load profiles, real time market interaction, demand response and other automated services. Service continuity will be improved. Customers may have the opportunity to select, and pay for, the level of power quality they need. System operation, customer service, maintenance planning, market functions, and system analysis and system planning will be integrated into an information technology infrastructure.

\section{Intentional controlled islanding.}

During severe system disturbances, large transmission systems will break up into preplanned islands where load and generation are balanced. As DER becomes more integrated into the distribution system, it will be possible to break up the distribution system into islands that are also self regulating, providing extremely high levels of power quality to critical loads. These islands may be as small as $10 \mathrm{MW}$ of load, or as large as 


\section{DRAFT}

several hundred MW. The islands could be pre-planned, and instituted as a result of potential contingencies, or they could be developed in real time using a set of algorithms that decide the best system configuration for any post contingency set of available circuits and generation. [2]

\section{Flexibility to deal with contingencies.}

During a contingency, the number of alarms coming from the distribution system can become unmanageable very quickly. In some systems, 500 alarms per minute can be generated in a simple perturbation. [3] The local agent can be instrumental in dealing with even large scale contingencies, because the small scale response to certain combinations of sensed variables will often be the same whether it is a large scale or small scale contingency. As discussed later, local agents will also be able to communicate rapidly with each other to minimize outage duration and speed recovery.

\section{Energy storage and power electronics}

Energy storage, combined with power electronics, can seamlessly deliver power to sustain normal operation during voltage sags and dips, and provide an uninterruptible power supply to a critical process system. Some of the key components of the needed energy storage systems will be batteries, ultra capacitors, flywheels, superconducting magnetic energy storage, and dc buses connecting to other devices. These will provide short- and long-term energy storage.

These devices will provide power to the distribution system through a power electronics interface and will provide dynamic voltage support capability and a host of other features. The interface will enable ancillary services (reliability services) such as reactive power compensation and frequency and voltage regulation. The interfaces will be able to adapt a wide variety of DER and energy storage devices to the distribution system. These power electronic interfaces will be developed to lower cost, improve reliability, and increase power and voltage levels. New types of power electronic switching devices will enable switching of higher current levels and higher voltages at lower costs.

\section{DER Integration}

Densely populated urban areas often use a network type of distribution system. In this system, a matrix or web of conductors is installed under the city streets and side walks. The network is typically fed from five to fifteen distribution feeder circuits. Sometimes more than 2 or 3 of these circuits can be lost before the network has to be shut down. Because of this redundancy, network reliability is already quite high. However, DER can be used to provide power quality in large buildings in networked areas, and to even operate the buildings in islanded mode when required. DER can also be used to improve the reliability of the network, and to improve the power factor of the network load. In addition, networks may someday be considered as microgrids - an aggregation of loads and generation that can either be operated independently or connected to the grid. 


\section{DRAFT}

In rural areas, DER can provide a significant service by reducing losses in long feeders and performing voltage regulation. Reactive power supplied at the load has a much greater impact than capacitor banks installed at substations. In addition, the effect of the reactive power supplied by capacitors decreases with the square of the voltage, but reactive power supplied by a generator actually becomes more effective as voltage droops.

In many areas of high load growth, because of the difficulty in obtaining the large parcels of real estate required for conventional generation, and because new generation is required quickly, DER will be the only practical option. In the next five to ten years, DER may constitute $20 \%$ or more of the nation's new generating capacity. Even at much smaller percentages - say 2 or 3\% - DER must be capable of supplying the reliability services presently provided by large turbine generators, such as spinning reserves, reactive power supply, and voltage and frequency regulation. It will not be desirable to disconnect DER from the grid when there is a disturbance if DER comprises 2 or 3\% of the generation. Instead, DER will remain connected and will help stabilize the system.

DER have small or no rotational inertia, and their characteristics are very different from those of large turbine generators. However, when DER are equipped with a power electronics interface, energy storage, and intelligent control systems, they will be capable of supplying a wide range of reliability services that exceed the capabilities of conventional large turbine generators.

The biggest complaint utility engineers have about DER is that it is a control and protection nightmare. This was true in the days of conventional distribution system control, but with local control agents, DER will be an integral and valuable player in distribution reliability.

\section{Looking to the Future}

Methods and design protocols should be prepared now so that distribution system modifications being made now will dovetail with the future distribution system. The urban distribution systems being used in many parts of the country were built in the 1950 's and 60's during a period of tremendous growth. Urban systems are now in a rather aged state, and repairs are expensive. Underground feeder cables, especially, are reaching the end of their useful lives. It is important to plan carefully when making modifications and upgrades to systems to ensure that systems are upgraded in the most cost effective manner possible, and to ensure that the modifications dovetail with the distribution system of the future. Utilities may want to improve their worst performing feeders first, or provide high quality service to key accounts. A feeder upgrade and management strategy could be developed which ensures that modifications will be compatible with future data acquisition and control methods.

\section{Areas Ripe for Technology Development}




\section{DRAFT}

Power electronics, static switches and FACTS

At least one author states that static interruption devices can have a total elapsed time from fault occurrence to interruption of less than $1 \mathrm{msec}$. [4] This means that voltage sag at the connected loads due to distribution system faults could be prevented. In addition, power electronics or Flexible AC Transmission Systems (FACTS) can provide a controlled level of reactive power support to the local distribution system for voltage regulation, and can provide the interface for a wide variety of DER and energy storage devices. With this interface, the local power quality level may be maintained at whatever level the customer desires.

\section{High Temperature Superconductivity}

The widespread use of superconducting materials in transmission and distribution has been precluded by the cost required to achieve the very low temperatures of liquid hydrogen. New materials have been found that are superconductive at temperatures of liquid nitrogen, a temperature far easier to achieve and far less costly than liquid hydrogen. Transformers and underground cable are now being developed for commercialization in the near term future, perhaps in three years. High temperature superconductivity promises great improvements in both efficiency and in the space required for substations and power transmission and distribution.

\section{Fault detection using current signatures and fault current control}

Arcing fault detection is now available in 120 volt devices, and this technology may soon be applied at the distribution level. Distribution faults are now interrupted based only on an assessment of the magnitude, distance and type of fault (single phase, double line to ground, etc.) Low current faults, such as arcing faults, or high resistance ground faults, are difficult to detect. In the future, methods such as signal processing analysis of current for characteristic signatures of faults will be used to detect and isolate these fault types.

The methods for fault detection and the clearing time for faults will be greatly improved through the use of an array of current and voltage sensors. Fault detection and location algorithms will enable the sensors to function together to dramatically reduce the number of customers impacted by fault clearing.

\section{Responsive load}

Responsive load will be key to improved levels of reliability and healthy electricity market operation. At the present time, there is very little elasticity of demand in the power market. When contingencies occur, such as the loss of transmission lines or generation, and shortages develop, the price for energy will rise in the wholesale market. In almost any other market system, this rise in price is met with a reduction in demand. In the present electricity market, most customers do not see the real time price. This 


\section{DRAFT}

means that demand does not decline and wholesale prices spike. That is what occurred in the energy crises in the Midwest and California in 2000 and 2001. Only a small percentage of customers need to respond to price, perhaps only 5 to $7 \%$, to have a healthy market system.

To date, load has not been able to respond to price because the communication and market systems did not exist. The market signals may be handled in the future over the internet, and the customer may have a website where they can plug in their strike price for air conditioning curtailment, for example. If they are away on a business trip, they may make the strike price quite low; if they are hosting a dinner party, they may make it quite high. The local system operator would have a statistical understanding of the demand response, and would be able to forecast his load accurately. This capability is one of the key attributes for the distribution system of the future.

\section{Communication and control}

The existing communication system that many utilities have for distribution automation is based on an 800 or $900 \mathrm{MHz}$ wireless system, and the number of channels available for future communication needs are limited. The wireless network will be used for communications from the central control authority to a limited number of field components and to field crews; it could also be used for paging for load control. The future, especially for urban systems, may be a fiber optic network, although internet technology is another option. Because utilities presently own the poles and ductwork, much of the infrastructure for installing a fiber optic system is already in place. In fact, utilities could lease portions of the fiber capacity to a third party, or include a high speed link in the residential user's package of services. The future utility communication network will include not only the system automation, but also the business information, billing, customer service and voice applications.

Utility Communications Architecture (UCA) was developed by EPRI to specify a suite of protocols that meet the requirements of the electric utility industry. UCA uses both Open Systems Interconnection standards and internet protocols. In theory, the advantage of using UCA on a project is the reduction in cost to integrate devices supplied by different vendors. However, there are other protocols that may have more common vendor acceptance. In addition, the ability to use an off the shelf browser to have a direct connection to field devices may present a security threat.

An unfortunate aspect of communication systems is that as they evolve, complexity increases. Many of the advanced protocols, while they are more flexible and allow greater communications control, also have more communications overhead. They require more bandwidth and greater speed due to their complexity. It is also a misconception that all devices that use the same protocol must work together. Depending on the types of monitoring and control desired, a complex protocol may not be necessary.

Real time local state estimation modeling 


\section{DRAFT}

In some urban areas and in load pockets near urban areas, load growth over the last few years has been much higher than predicted. This surprising growth is due not only to increasing use of the Internet and communications systems, but also to the rapid growth of conventional loads. To ensure overall quality of service, a "predictive assessment will need to be made that includes the distribution system's operating state, a comparison against on-going forecast and analysis of potential future events, and course correction to avoid problems and remain within an acceptable zone of reliability, all in real-time." [5]

Some of the capabilities the energy delivery management system may have are:

- Provide an accurate representation of current state and loading of feeders and components.

- Derive equipment ratings dynamically.

- Track the system "trajectory" to identify potential difficulties.

- Identify areas currently in need of attention such as overloaded equipment, inadequate voltage, and phase imbalance.

- Recommend and prioritize corrective actions in time frame of hours or longer and takes corrective actions in time frames of seconds or minutes.

- Assess reliability of delivery based on a probability analysis using real time data.

- Establish options for power purchases based on demand and forecast.

\section{Local independent agents}

De-centralized intelligence offers one especially attractive benefit - the communication system can operate at a much slower speed. Routine status information to the central controller will most likely be updated only once every several minutes. The local agent will act independently for many tasks such as clearing and isolating faults, reconfiguring the local system, or regulating voltage. Other tasks will be at the direction of the central controller. Prioritizing information flow will be the first step in system design. During a system disturbance, the local agent will be authorized to act rapidly to address problems.

\section{System flexibility and adaptability to contingencies}

Distribution system automation has already taken great strides in providing higher levels of reliability through such designs as loop feeders which determine the location of the fault, isolate the fault, and then feed the load through an alternate path. The entire operation is controlled locally and happens quickly. Dealing with major contingencies on the distribution level though is still relatively unknown.

Post contingency operation can be rather complex, complicated by the fact that restoration following an outage can lead to unusually high load, especially during heating or cooling seasons. Transformer capacity can be stressed, and restoration must be modeled based on equipment remaining in service, duration of outage, expected cold load pickup factors, load priorities and a number of other factors to optimize recovery time. 


\section{DRAFT}

A real time system model will be required to understand the load that will be presented, and to calculate the optimum restoration path.

\section{The Market, the Key to the Future}

\section{Financial Incentive}

DER and responsive load will become widespread across the nation once there is a strong financial incentive for both the owner and for the utilities. These financial incentives will not exist until there is a market for the services the microgrid and EMS can provide to the distribution system. The development of such a market will take major institutional changes, but the reforms are occurring already in some parts of the nation. Services such as voltage regulation are being considered in trial programs in some areas.

Of further encouragement is the fact that FERC is encouraging open competitive markets for generation for both energy and ancillary services. FERC ordered the unbundling of ancillary services from transmission to promote competitive markets and is pursuing standard market design, which should improve economic efficiency and lower electricity prices. Beyond the argument of fairness, having DER and loads participate as suppliers, as well as consumers, of electricity services improves resource utilization.

\section{Local Supply of Services to the Distribution System}

DER may take the form of an aggregation of electrical loads and generation. The generators may be fuel cells, microturbines, reciprocating engines, or any of a number of alternate power sources. Often, the generators also supply heat for local needs such as space heating or dehumidification. An Energy Management System (EMS) will be used to make decisions regarding the best use of the generators for producing electric power and heat, as well the optimization of the energy used. These decisions will be based on the heat requirements of the local equipment, the weather, the price of electric power, the cost of fuel and other considerations.

There are a number of other functions the EMS can perform:

- Intelligent energy storage based on weather-related or process needs

- Full optimization of combined heating and power (CHP)

- Allowing generators to function without a dedicated generator control system since the EMS will dispatch only voltage and power

- Generator operation based on the energy market for both gas and electricity

- Optimization of HVAC through advanced control strategies

- Minimizing pollution based on algorithms that consider CHP and displaced emissions

- Enhanced power quality where, for example, a loss of grid power causes a seamless transfer to stand alone power involving only a loss of non-critical loads 


\section{DRAFT}

- Monitoring building conditions and responding to threats such as fire or chemical/biological attacks

- Supporting the grid by supplying an array of ancillary services, such as voltage regulation and reserve power

Most importantly, investments in DER and load management are not only beneficial to those consumers who use the technologies, they also lower the wholesale market prices paid by all consumers [6]. The Massachusetts Department of Energy Resources concluded that the measures installed in their post-restructuring efficiency program lowered the participants' electricity cost by $\$ 20$ million in 1999 . The Department also concluded that, by lowering peak demand at high cost periods, the programs provided reliability and power cost savings to all customers, participants and non-participants alike. The benefit in just 13 hours on one high-cost day exceeded $\$ 6$ million due to the effect on the wholesale energy-clearing price.

\section{Critical Market Issues}

Six issues that must be addressed for the DER and load to fully respond to market conditions are [7]:

1. Retail tariffs typically do not reflect wholesale costs. Consequently, DER and load are not price sensitive to electricity and natural gas costs, and do not respond.

2. Wholesale energy markets do not have a built in demand side that accommodates responsiveness from microgrids. DER and load, equipped with EMS, could provide a highly elastic demand curve. This would be of significant value in a day-ahead market, where the EMS could bid in "capacity" based on anticipated weather and process plans.

3. Load profiles used to assign wholesale costs to load serving entities fail to account for the actual costs of service. A level profile has a significantly lower actual cost. A highly fluctuating profile may create a regulation burden.

4. Demand side resources are effectively excluded from the wholesale markets for reliability services. The ancillary service markets should be opened to DER and load.

5. Transmission constraints are often not recognized in the pricing of wholesale or retail energy services. Locational pricing reveals the value of distributed resources in alleviating congestion across constrained interfaces.

6. The markets for reactive load management, voltage regulation, and other demand side services are immature, if they exist at all, and will remain uncertain until equitable market rules are established. There is a need to reform price caps and default service rates to reflect the true value of load profile response at different times and locations.

As these changes are made, low cost, reliable technologies will evolve in power electronics, high temperature superconductivity, fault detection, responsive load and communications. The resulting improvements in system flexibility and safety will lead to 


\section{DRAFT}

lower overall cost and improved reliability, and greater power for the individual consumer to choose their own energy options.

\section{References}

1. Maintenance Scheduling in Restructured Power Systems, M. Shahidehpour, Kluwer Academic Publishers, Norwell, Massachusetts <kluwer@wkap.com>

2. "Modeling the Impact of Automation and Control on the Reliability of Distribution Systems" Ying He, IEEE 0-7803-6420-1/00

3. "An Agent Oriented Paradigm for the Design of Complex Systems: Energy Distribution Automation, an Application Domain" Joaquin Delgado, IEEE 0-7803-2672$5 / 95$

4. "Utilization of Static Circuit Breakers in Loop Distribution Systems for Reliability Improvement" A. Cali, IEEE 0-7803-5105-3/98

5. "The Rise of Energy Delivery Management Systems" K. I. Geisler, CES International, Plymouth, Minnesota, http://www.ces.com/index_plain.html

6. Richard Cowart, "Efficient Reliability, The Critical Role of Demand Side Resources in Power Systems and Markets", the Regulatory Assistance Project, June, 2001, http://www.rapmaine.org/efficiency.html

7. "Efficient Reliability, The Critical Role of Demand Side Resources in Power Systems and Markets" Richard Cowart, The Regulatory Assistance Project, June, 2001, http://www.rapmaine.org/efficiency.html 